\title{
An 18 Alanine Repeat in a Severe Form of Oculopharyngeal Muscular Dystrophy
}

\author{
L. Jouan, D. Rocheford, A. Szuto, E. Carney, K. David, P.A. Dion, G.A. Rouleau
}

Can J Neurol Sci. 2014; 41: 508-511

Oculopharyngeal muscular dystrophy (OPMD) is an autosomal dominant adult-onset neuromuscular disease that usually develops in the fifth or sixth decade of life and which is characterized by progressive ptosis, dysphagia and proximal limb weakness as well as a positive family history ${ }^{1}$. The disease is usually inherited with complete penetrance and without gender preference ${ }^{1}$. It is caused by trinucleotide repeat expansions that result in a lengthened polyalanine tract in the polyadenylate binding protein nuclear-1 gene ( $P A B P N 1)$, located on chromosome 14q11.2-q13. These polyalanine expansions referred to as $(\mathrm{GCN})_{\mathrm{n}}$ are believed to arise from unequal allelic homologous recombination during meiosis and/or mitosis ${ }^{2}$. The wild type allele of the $P A B P N 1$ gene harbors 10 alanine codons that are located immediately downstream to the start codon in exon 1 where they code for an homopolymeric stretch of 10 alanine residues, also referred as $(\mathrm{GCN})_{10}$. Mutant dominant $P A B P N 1$ alleles underlying OPMD range between 12 and 17 $(\mathrm{GCN})$ repeats. A $(\mathrm{GCN})_{11}$ polymorphism has been reported in North America, Europe and Japan but it does not lead to OPMD or another specific phenotype when in an heterozygous state ${ }^{2}$. However, rare cases of $(\mathrm{GCN})_{11}$ homozygous carriers have been reported and, by comparison to individuals with $(\mathrm{GCN})_{12-17}$ alleles, they present with relatively mild OPMD symptoms and at a later age of onset (sixties). To date, PABPN1 is the only known gene to be associated with OPMD.

Interestingly, in our study based on 72 French-Canadian families, we have shown that $5 \%$ of familial cases had a $(\mathrm{GCN})_{12}, 40 \%$ a $(\mathrm{GCN})_{13}, 26 \%$ a $(\mathrm{GCN})_{14}, 21 \%$ a $(\mathrm{GCN})_{15}, 7 \%$ a $(\mathrm{GCN})_{16}$ and finally $1 \%$ a $(\mathrm{GCN})_{17}$ PABPN1 allele ${ }^{3}$. A relationship between the length of the $(\mathrm{GCN})$ repeat and symptom severity was found because compound heterozygote carriers of $(\mathrm{GCN})_{11}(\mathrm{GCN})_{13}$ alleles displayed a more severe phenotype than siblings who were compound heterozygous carriers of $(\mathrm{GCN})_{10}(\mathrm{GCN})_{13}$ alleles or homozygous siblings with $(\mathrm{GCN})_{11}$ alleles $^{3,4}$. In that vein, Hill et al observed an earlier age of onset for individuals with longer repeat expansions ${ }^{5}$. However, Tondo and colleagues reported a range of symptom severity among carriers of a mutated $(\mathrm{GCN})_{n}$ of the same size, while Semmler and colleagues reported autosomal recessive OPMD cases harboring $(\mathrm{GCN})_{11}$ alleles with severe phenotypes ${ }^{6,7}$. Therefore, it has been suggested that additional genetic and environmental factors could modulate disease expression and consequently, the correlation between severity and the polyalanine expansion size is still a subject of debate. Here, we report a family with two affected sisters who are both carriers of an unusual expansion of 18 polyalanines in $P A B P N 1$, the longest $(\mathrm{GCN})_{\mathrm{n}}$ expansion reported to date. We believe this severe and early-onset OPMD phenotype correlates with the size of the expanded mutated allele.

\section{Clinical report}

The first patient is a 47 year-old female who was diagnosed at approximately age 7 with rheumatic fever and Sydenham's chorea with intermittent exacerbations (Figure 1a). She graduated from college and was socially very active. She held a steady job in the past. She reportedly had unilateral ptosis and asymmetry of her right eye from birth and nasal speech that worsened with time. The patient had difficulty with her balance, especially on stairs. Magnetic resonance imaging (MRI) of the brain in July 2012 revealed a solitary focus of T2 hyperintensity within the white matter of the right frontal lobe just anterior and slightly superior to the insular cortex. There was no corresponding diffusion-weighted abnormality nor was there mass effect. The finding was considered entirely non-specific. The remainder of the brain MRI was within normal limits. In July 2012, there were no swallowing problems by her report; she ate all foods and had not changed her diet. She complained of lethargy and no longer worked. She had a history of severe depression that led to hospitalizations in 2011 and subsequently in 2013 due to suicidal thoughts. On neurological examination in August 2013 she had a slow nasal speech with mild dysarthria, bilateral facial weakness, and bilateral ptosis. Her arms and legs were noted to be thin, but she had full strength throughout. While she had not been formally evaluated, she was reportedly starting to have memory problems as of November 2013.

The second patient is her sister, a 48 year-old woman who had a history of mental illness since her early 30s. She was hospitalized for two weeks at age 38 for paranoia. She became severely agoraphobic. She had progressive muscle weakness and was diagnosed with a neuromuscular disorder at approximately age 41-42 (Figure 1a). A foot drop was observed, but

From the Montreal Neurological Institute and Hospital (LJ, DR, AS, PAD, GAR) Department of Medicine, McGill University; Centre de Recherche du Centre Hospitalier de l'Université de Montréal (CRCHUM) (LJ, DR, AS, PAD), Department of Pathology and Cell Biology (PAD), Faculty of Medicine, Université de Montréal, Montreal, Quebec, Canada; New York Methodist Hospital (EC, KD), Department of Medicine, Division of Genetics, Brooklyn; Metropolitan Hospital Center (KD),

Department of Pediatrics, Genetic Services, New York, New York, USA. Received September 23, 2013. Final Revisions Submitted February 10, 2014. Correspondence to: Guy Rouleau, Montreal Neurological Institute and Hospital, 3801 University St., Room 636, Montreal, Quebec, H3A 2B4, Canada.

Email: guy.rouleau@mcgill.ca. 


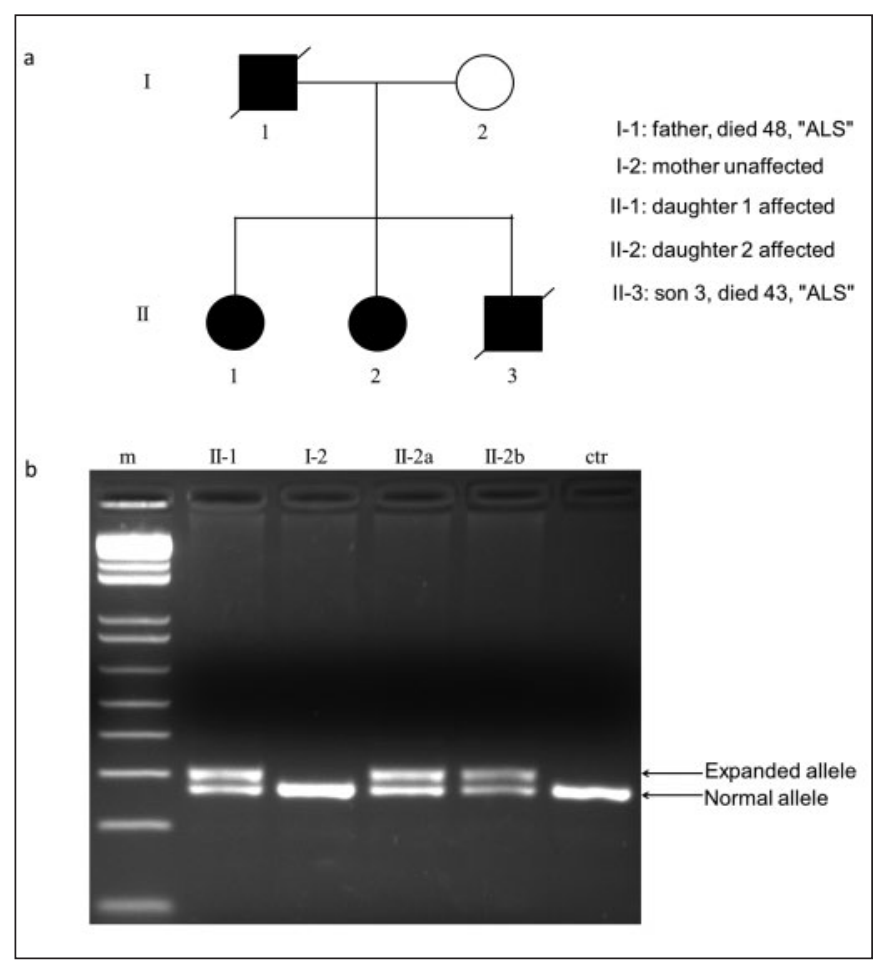

Figure 1: (a) Pedigree of the oculopharyngeal muscular dystrophy (OPMD) family. Filled symbols indicate affected and open symbols indicate unaffected family members, (b) PCR products obtained by PCR amplification on genomic DNA from blood sample obtained from affected daughter 1 (lane II-1), affected daughter 2 repeated in duplicate (lanes II-2a and II-b), unaffected mother (lane I-2) and unrelated control (lane ctr) by using oligonucleotide primers located in PABPN1 exon 1. Arrows indicate the expanded and normal alleles.

electromyogram (EMG) was not performed due to patient discomfort. Progressive swallowing problems developed around age 44-45 along with nasal, mildly dysarthric speech. She had memory problems and sleep disturbances. On neurology evaluation in 2010 , she was unable to recall any of three words in five minutes, she performed poorly on attention/concentration task and naming and she scored 40/57 on a modified mental status exam and in the conversation context. She presented with psychomotor delays, difficulties synthesizing linguistic information and intermittent reduced comprehension for simple questions. On physical examination in 2011, bilateral eyelid ptosis, open mouth, mildly limited range of motion of eyes laterally and limited upward gaze were noted. Reevaluation in 2013 by neurology confirmed a nasal voice and bilateral ptosis. Extraocular movements were considered intact. There was bifacial weakness, weak tongue, foot drop and mild weakness of the triceps and left quadriceps with mild weakness of finger flexion and extension. As of November 2013, she became completely incontinent. Both sisters continued to lose weight and neither was able to open a bottle of water on their own.

The patients' brother was diagnosed at age 12 with a neuromuscular disorder. In his mid-30s he developed eyelid ptosis, difficulty swallowing, and dystonic movements. He was also reported to have had mental illness, suicidal thoughts and heavy drug use, which included heroin daily beginning at approximately age 18. Neurology evaluations from 2003-2004 indicated dysphagia requiring gastrostomy tube feedings, dysarthria, facial weakness, mild head titubation, and saccades moderately slow in all directions. There was muscular atrophy without weakness, mild chorea of the trunk and limbs while seated with chorea distally in both upper extremities blending with dystonic movements while he walked. Cognition was said to be unimpaired. He died at age 43 from suspected amyotrophic lateral sclerosis (ALS). Genetic testing was performed for Huntington's disease ( $H D$ gene expansion), ALS (SODI gene sequencing) and spinocerebellar ataxia types 1-3 (SCA 1-3 gene expansion). No mutation was detected.

The father of all three patients was diagnosed in his 30s with paranoid schizophrenia, progressive eyelid ptosis, and myasthenia gravis. He died at approximately 50 years-of-age from supposed ALS. Mental illness was also reported for the paternal grandmother and paranoid schizophrenia for the maternal grandmother. The paternal family is Ashkenazi Jewish from Russia (region unknown) and the maternal family is Ashkenazi Jewish from Russia and Hungary. Consanguinity was denied.

We performed polymerase chain reaction (PCR) amplifications on DNA samples from the two affected sisters and the unaffected mother using oligonucleotide primers located in PABPN1 exon 1. Polymerase chain reaction products were loaded on agarose gel where two bands of different size were observed for both sisters (Figure 1b, lanes II-1 and II-2a and IIb) in contrast to the mother (Figure 1b, lane I-2) and an unrelated control (Figure 1b, lane ctr). Sanger sequencing revealed a heterozygous polyalanine expansion of $18(\mathrm{GCN})$ repeats that consists of ATG (GCG) ${ }_{6}(\mathrm{GCA})_{1}(\mathrm{GCG})_{7}(\mathrm{GCA})_{3}$ GCG GGG (Figure 2a and b) encoding for Met-(Ala) ${ }_{18}$-Gly in both sisters (Figure 2c) whereas the mother harbored the normal allele ATG $(\mathrm{GCG})_{6}(\mathrm{GCA})_{3}$ GCG GGG encoding for Met-(Ala) $)_{10}$-Gly (Figure $2 \mathrm{~b}$ and $\mathrm{c}$ ). Unfortunately, DNA samples from the brother and the father were not available.

\section{DisCUSSION}

We report a family with OPMD with severe phenotypes and possibly a very early age of onset. We have shown that both sisters have an expanded $(\mathrm{GCN})_{18}$ polyalanine tract in the PABPNl gene in the heterozygous state that consists of (GCG) $(\mathrm{GCA})_{1}(\mathrm{GCG})_{7}(\mathrm{GCA})_{3} \mathrm{GCG}$ whereas the normal allele in the mother consists of (GCG) ${ }_{6}(\mathrm{GCA})_{3} \mathrm{GCG}$; the father is likely an obligate mutation carrier. Extra triplet repeats in OPMD were initially described as pure $(\mathrm{GCG})_{n}{ }^{3}$, while several subsequent studies revealed the presence of (GCA) interspersed within the expanded $(\mathrm{GCG})_{\mathrm{n}}$ repeats. In our case, the exact mechanism that led to $(\mathrm{GCA})_{1}(\mathrm{GCG})_{7}$ repeat is unknown but could be similar to what has been observed with the previously described (GCG) ${ }_{13}$ allele where an addition of seven sequential (GCG) triplets are attributed to slippage or a combination of unequal cross-over and slippage as described by Robinson $e t a l^{2}$.

The unprecedented length of this polyalanine stretch could explain the severity and the early age of onset of the disease in this family and supports a correlation between the size of the expansion and the severity of the disease. Oculopharyngeal muscular dystrophy is considered a late-onset disease with the 


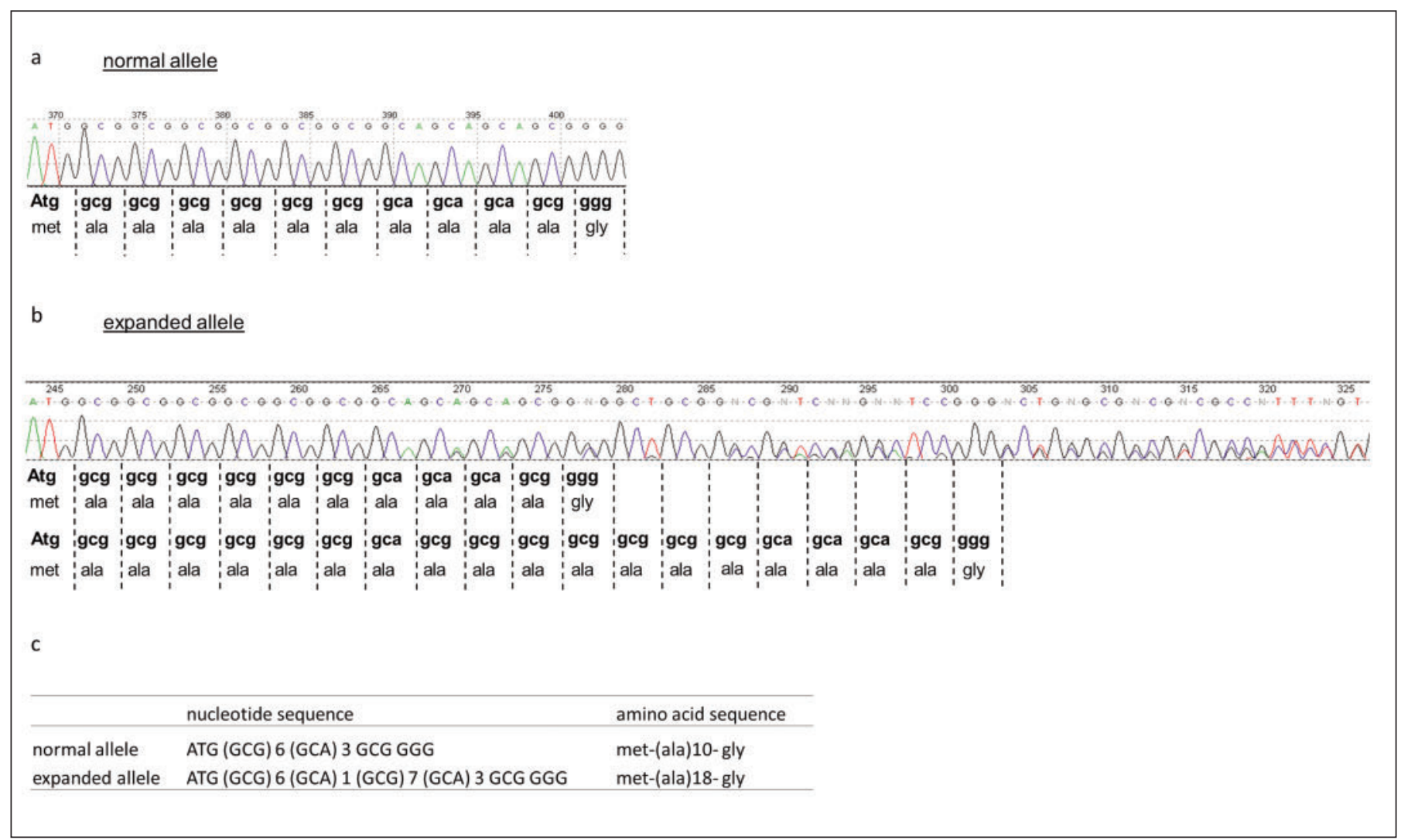

Figure 2: PABPN1 normal and expanded alleles observed in the unaffected mother and her two OPMD affected daughters, respectively. (a) Sequence trace of the normal polyalanine repeat located in the first exon of the PABPN1 gene of the mother and the unrelated control, $(b)$ expanded allele harboring 18 polyalanine repeats observed in the two affected daughters, (c) resulting nucleotide and amino acid sequences identified in the normal and expanded alleles.

majority of symptoms usually starting after 45 years of age. Brais et al, reported mean ages of 48.1 and 50.7 years for ptosis and dysphagia respectively in a cohort of French-Canadians with a $(\mathrm{GCN})_{13}$ mutation, which is consistent with a study in a cohort of Hispanic New Mexican cases with dominant OPMD and mean age of onset of 52 and 54 for ptosis and dysphagia ${ }^{3,8}$. However, the severity of the dominant OPMD phenotype is variable and severe cases with symptoms of ptosis and dysphagia before 45 years of age and incapacitating proximal leg weakness before 60 years of age represent 5 to $10 \%$ of all cases. It is believed that the variability of age of onset and severity of weakness may depend in part on the size of the expansion; however, this important issue is still unresolved. Interestingly, individuals who are homozygous for an autosomal dominant OPMD mutation present a more severe disease such as demonstrated in a study with four French-Canadian and three Bukhara Jewish patients with an average age of onset 18 years earlier than heterozygotes for the same mutant allele ${ }^{3,9,10}$. It was also shown that clinical features in these patients are early manifestations of the classical signs plus some unusual features such as slow and paretic eye movements. Proximal weakness in lower limbs, a very late presenting feature in heterozygotes, occurred in seven homozygous individuals before 51 years of age $^{9}$.
Cognitive and psychiatric impairments were prominent in all affected members of our family with an expanded $(\mathrm{GCN})_{18}$ polyalanine tract. Interestingly, Blumen et al reported cognitive decline and psychotic manifestations in four French-Canadian and six Uzbek Jewish homozygous $(\mathrm{GCN})_{13}$ allele carriers ${ }^{11}$. Later on, neuropsychological evaluation of 11 OPMD heterozygote patients (with GCN expansion ranging in size from 13 to 16 repeats) revealed some cognitive impairments and psychological disorders with a negative correlation between the expansion size and some of the neuropsychological scores ${ }^{12}$. Altogether, this strongly suggests that behavioral and psychological disturbances correlate with the expansion size in heterozygote OPMD patients and seem to be more severe and show a more rapid progression with respect to this expansion size in homozygous and heterozygous carriers.

We cannot be certain that the fatal disease seen in the father and brother was caused by the $(\mathrm{GCN})_{18}$ expansion. He and his son had a reduced lifespan; we can speculate that their unusual presentation did not allow for an accurate diagnosis, resulting in what was considered to be possible "ALS". They certainly had the typical OPMD ptosis and dysphagia at an early age.

Our study supports the hypothesis that OPMD patients with longer expansion may progress more rapidly and present additional clinical features such as cognitive symptoms that are 
usually absent from the typical OPMD with $(\mathrm{GCN})_{12-17}$ mutated alleles. Studies in additional OPMD cohorts should help answer this important issue.

\section{ACKNOWLEDGEMENTS}

The authors thank the family involved in their study and the referring neurologist, Dr. Miran Salgado.

\section{REFERENCES}

1. Abu-Baker A, Rouleau GA. Oculopharyngeal muscular dystrophy: recent advances in the understanding of the molecular pathogenic mechanisms and treatment strategies. Biochim Biophys Acta. 2007;1772(2):173-85.

2. Robinson DO, Hammans SR, Read SP, Sillibourne J. Oculopharyngeal muscular dystrophy (OPMD): analysis of the PABPN1 gene expansion sequence in 86 patients reveals 13 different expansion types and further evidence for unequal recombination as the mutational mechanism. Hum Genet. 2005;116(4):267-71

3. Brais B, Bouchard JP, Xie YG, et al. Short GCG expansions in the PABP2 gene cause oculopharyngeal muscular dystrophy. Nat Genet. 1998;18(2):164-7.

4. Hebbar S, Webberley MJ, Lunt P, Robinson DO. Siblings with recessive oculopharyngeal muscular dystrophy. Neuromuscul Disord. 2007;17(3):254-7.
5. Hill ME, Creed GA, McMullan TF, et al. Oculopharyngeal muscular dystrophy: phenotypic and genotypic studies in a UK population. Brain. 2001;124(Pt 3):522-6.

6. Semmler A, Kress W, Vielhaber S, Schroder R, Kornblum C. Variability of the recessive oculopharyngeal muscular dystrophy phenotype. Muscle Nerve. 2007;35(5):681-4.

7. Tondo M, Gamez J, Gutierrez-Rivas E, Medel-Jimenez R, Martorell L. Genotype and phenotype study of 34 Spanish patients diagnosed with oculopharyngeal muscular dystrophy. J Neurol. 2012;259(8):1546-52.

8. Becher MW, Morrison L, Davis LE, et al. Oculopharyngeal muscular dystrophy in Hispanic New Mexicans. JAMA. 2001; 286(19):2437-40.

9. Blumen SC, Brais B, Korczyn AD, et al. Homozygotes for oculopharyngeal muscular dystrophy have a severe form of the disease. Ann Neurol. 1999;46(1):115-18.

10. Blumen SC, Sadeh M, Korczyn AD, et al. Intranuclear inclusions in oculopharyngeal muscular dystrophy among Bukhara Jews. Neurology. 1996;46(5):1324-8.

11. Blumen SC, Bouchard JP, Brais B, et al. Cognitive impairment and reduced life span of oculopharyngeal muscular dystrophy homozygotes. Neurology. 2009;73(8):596-601.

12. Dubbioso R, Moretta P, Manganelli F, et al. Executive functions are impaired in heterozygote patients with oculopharyngeal muscular dystrophy. J Neurol. 2012;259(5):833-7. 